\title{
Dexterity of underactuated six degrees of freedom three dimensional redundant manipulators
}

\author{
Samer Yahya ${ }^{1} \quad$ Haider Abbas F. Mohamed Almurib ${ }^{2}$ \\ M. Moghavvemi ${ }^{3}$
}

(Received 23 November 2013; revised 11 August 2014)

\begin{abstract}
We quantify the dexterity of underactuated robot manipulators equipped with active and passive joints. We discuss the harnessing of redundant degrees of freedom in a robotic manipulator in order to keep it in service. We assume that the passive joints are locked at an arbitrary known position. There are three important dexterity measures: workspace volume, reachability, and manipulability. We discuss the workspace volume of a six degrees of freedom, three dimensional, redundant manipulator with an arbitrarily located passive joint.
\end{abstract}

http://journal.austms.org.au/ojs/index.php/ANZIAMJ/article/view/7709 gives this article, (C) Austral. Mathematical Soc. 2014. Published September 2, 2014, as part of the Proceedings of the 11th Biennial Engineering Mathematics and Applications Conference. ISSN 1446-8735. (Print two pages per sheet of paper.) Copies of this article must not be made otherwise available on the internet; instead link directly to this URL for this article. 


\section{Contents}

1 Introduction

C417

2 Workspace analysis

C419

3 Crippling of the manipulator

C423

3.1 Crippling of $\theta_{1}$ joint

C423

3.2 Crippling of $\theta_{2}$ joint

C424

3.3 Crippling of $\theta_{3}$ joint

$\mathrm{C} 426$

3.4 Crippling of $\theta_{4}, \theta_{5}, \ldots, \theta_{n}$ joint

C427

4 Simulation results

C429

4.1 Breakdown of $\theta_{1}$ joint . . . . . . . . . . . C430

4.2 Breakdown of $\theta_{2}$ joint . . . . . . . . . . . . C430

4.3 Breakdown of $\theta_{3}$ joint . . . . . . . . . . . . . C431

4.4 Breakdown of $\theta_{4}$ joint $\ldots \ldots \ldots \ldots \ldots$ C $\ldots \ldots 32$

4.5 Breakdown of $\theta_{5}$ joint $\ldots \ldots \ldots \ldots \ldots$ C432 . . . . . . . . .

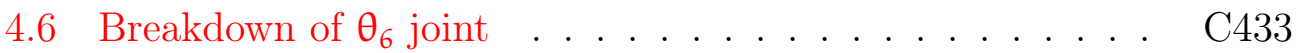

5 Conclusion

C434

References

C435

\section{Introduction}

The last two decades witnessed considerable progress in the study of underactuated robots, which have fewer working actuators than design degrees of freedom because of design failure. 'Underactuated' refers to the manipulator having fewer working actuators than joints [1]. Most often, a passive, or underactuated, joint results from a failure on a joint's mechanism, system, or alternative part. A passive joint might also be a design feature of a manipu- 
lator, as a hyper-redundant manipulator with more links than actuators may result in energy saving, price decrease, or weight reduction [2].

This article is based on the work of Yahya et al. [3], which presented a mechanical design for a three dimensional, planar, redundant manipulator, which guaranted to decrease the weight of the manipulator by decreasing the number of motors needed to control the manipulator. Yahya et al. studied the kinematics of the manipulator and showed it avoids singular configurations. Later Yahya et al. [4] explained the dynamics of this manipulator in detail and showed its ability to acheive joint torque minimization. Here, the method of dexterity explained by Bergerman and $\mathrm{Xu}[2]$ is used to show the dexterity of the manipulator studied by Yahya et al. [3, 4].

In order to study the kinematic dexterity of such mechanisms, we assume that the passive joints are locked, whereas the remaining (active) ones move freely. If a passive joint arises from a joint failure, then we assume that it is locked at the position where it failed by a high-level fault-detection system. We surmise that the positions at which the passive joints are locked are known, either because their position sensors still work reliably, or because other sensors are available. Bergerman and $\mathrm{Xu}$ [2] proposed an optimization index to find the angles at which the passive joints should be locked to maximize the dexterity of the underactuated manipulator. Pradeep et al. [5] suggested an alternative strategy for dealing with an uncontrollable or crippled joint. Commercial robots, which are designed to perform a wide range of tasks, often possess more degrees of freedom than are required for any particular work assignment. A judicious use of these redundant degrees of freedom may allow the disabled robot to remain in service pending repair, thereby dramatically enhancing the device's reliability. The proposed strategy is to monitor the joints continuously and, should one of them become crippled, apply a brake to immobilize it immediately. Then, if the desired destination is still attainable, devise in real time a new set of kinematic instructions which enable the remaining joints to bring the end-effector to the required position.

Roberts [6] and Roberts and Maciejewski [7] discussed the decrease in a 
manipulator's kinematic manipulability index and worst-case dexterity due to the failure and locking of one or more of its joints. They were concerned with finding pre-failure optimal configurations of the manipulator that guarantee that the post-failure manipulability is maximized. A common mode of robot failure is for one of the power sources or transmission mechanisms to become inoperable, resulting in a loss of control over one of the joints. Depending upon the details of the actuator and the nature of the failure, this may cause the joint to lock up or go limp. In neither case would the end-effector be able to reach its desired position and orientation. Indeed, the results could be catastrophic, particularly if the other joints continue to move as if there were no problem. The robot might, for instance, crash into some obstacle that it is normally programmed to avoid, or deposit a hazardous material at an inappropriate location. One obvious solution to the problem is to monitor the state of the various joint parameters and apply brakes to freeze the robot in its current state the moment a failure occurs. This would prevent the sort of uncontrolled motions described above. However, in the case of continuous flow production processes, the resulting disruption could lead to substantial losses of revenue. Moreover, if the robot is serving in some truly critical capacity, then the shutdown may itself prove to be life threatening. Accordingly, this article examines a crippled version of the manipulator designed by Yahya et al. $[3,4]$. The object is to determine how the robot behaves after losing the use of one of its joints. The first question to be addressed is whether or not the remaining joints afford enough mobility for the manipulator to continue operating and cover the entire workspace.

\section{Workspace analysis}

Suppose that joints $P_{1}, \ldots, P_{n_{p}}\left(\right.$ with $\left.n_{p}<n\right)$, are passive joints locked at an arbitrary position within their joint limits, and $n$ is the number of degrees of freedom when all the motors are operable. For convenience we represent the passive set of joints by $I_{p}$, and the active and passive joint rotations by 
$\theta_{\mathrm{a}}$ and $\theta_{\mathrm{p}}$, respectively.

Being able to access to a large number of points in Cartesian space is generally a design benchmark and a desirable attribute of a robot manipulator. When the manipulator has both active and passive joints, it is desirable that the number of reachable points (which create the reachable workspace) is as large as possible. Denote by $\mathrm{V}$ the volume or area of a fully actuated manipulator's workspace, and by $V_{I_{p}}$, that of the corresponding underactuated manipulator. Whereas $V$ is a fixed quantity, $V_{I_{p}}$ depends on the positions where the passive joints are locked. The relative workspace loss is

$$
\tilde{V}_{I_{p}}\left(\theta_{p}\right)=1-\frac{V_{I_{p}}\left(\theta_{p}\right)}{V} .
$$

To control the motion of the end-effector of the manipulator shown in Figure 1(a), all the motors of the manipulator should be controlled. For example, to command a five links planar redundant manipulator with the ability to rotate around its vertical axis, the six motors (five motors for each joint bend and one motor to rotate the whole manipulator around its upright axis) of the manipulator ought to be controlled. Using our previously published method $[7,8]$, the configuration of the manipulator has three controllable angles instead of $(n+1)$ angles. Figure $1(b)$ shows the configuration of the manipulator when there are just three controllable angles.

Because the end-effector follows any desired path by controlling three angles $\left(\theta_{1}, \theta_{2}\right.$ and $\left.\theta_{3}\right)$ only, instead of using a motor for each joint angle only three motors are used to control the manipulator. In this manipulator, even though the manipulator has six degrees of freedom, there are only three active joints while the other joints are passive. Figure 2 shows the mechanism of this manipulator. Samer et al. [3, 4] provided more details.

For the manipulator shown in Figure 1(a), the position coordinates of the 

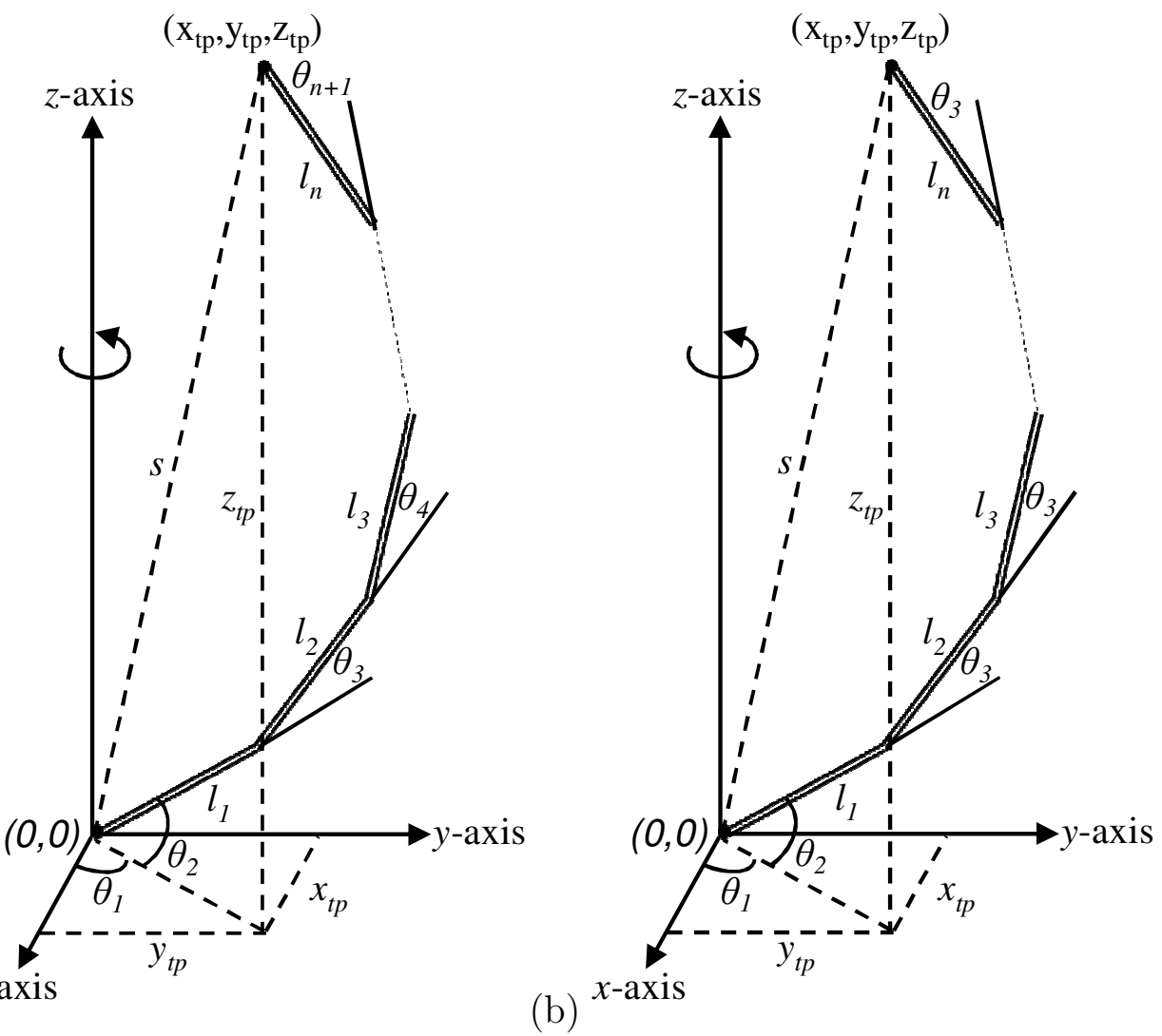

(a) $x$-axis

b) $x$-axis

Figure 1: (a) A three dimensional planar redundant manipulator configuration; (b) a three dimensional planar redundant manipulator configuration using the method of Yahya et al. [7, 8]. 

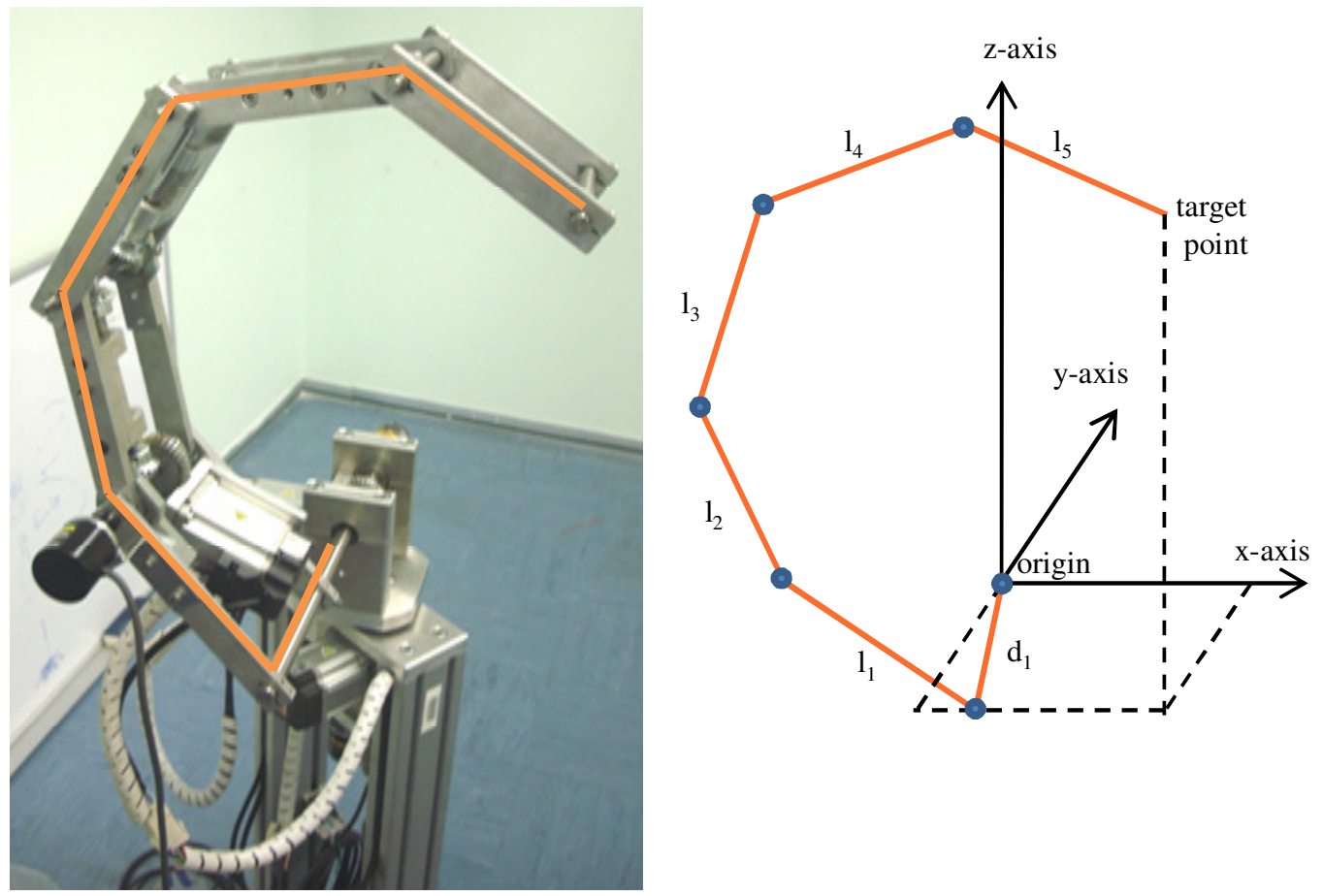

Figure 2: The manipulator used in experiments.

target point are

$$
\begin{aligned}
& x_{\text {tp }}=\cos \theta_{1}\left[l_{1} \cos \left(\theta_{2}\right)+l_{2} \cos \left(\theta_{2}+\theta_{3}\right)+\cdots+l_{n} \cos \left(\theta_{2}+\theta_{3}+\cdots+\theta_{n+1}\right)\right], \\
& y_{\text {tp }}=\sin \theta_{1}\left[l_{1} \cos \left(\theta_{2}\right)+l_{2} \cos \left(\theta_{2}+\theta_{3}\right)+\cdots+l_{n} \cos \left(\theta_{2}+\theta_{3}+\cdots+\theta_{n+1}\right)\right] \\
& z_{\text {tp }}=l_{1} \sin \left(\theta_{2}\right)+l_{2} \sin \left(\theta_{2}+\theta_{3}\right)+\cdots+l_{n} \sin \left(\theta_{2}+\theta_{3}+\cdots+\theta_{n+1}\right),
\end{aligned}
$$

where $n$ is the number of links, the links are of length $l_{1}, \ldots, l_{n}$ and make angles $\theta_{1}, \ldots, \theta_{n+1}$. For the manipulator shown in Figure $1(b)$ using the method of Yahya et al. [8], the angles between the links are equal, $\theta_{3}=\theta_{4}=$ 
$\cdots=\theta_{\mathfrak{n}+1}$. Therefore, the position coordinates of the target point are

$$
\begin{aligned}
x_{\mathfrak{t p}} & =\cos \theta_{1}\left[l_{1} \cos \left(\theta_{2}\right)+l_{2} \cos \left(\theta_{2}+\theta_{3}\right)+\cdots+l_{n} \cos \left(\theta_{2}+(n-1) \theta_{3}\right)\right], \\
& =\cos \theta_{1} \sum_{k=1}^{n} l_{k} \cos \left[\theta_{2}+(k-1) \theta_{3}\right], \\
y_{\mathfrak{t p}} & =\sin \theta_{1}\left[l_{1} \cos \left(\theta_{2}\right)+l_{2} \cos \left(\theta_{2}+\theta_{3}\right)+\cdots+l_{n} \cos \left(\theta_{2}+(n-1) \theta_{3}\right)\right], \\
& =\sin \theta_{1} \sum_{k=1}^{n} l_{k} \cos \left[\theta_{2}+(k-1) \theta_{3}\right], \\
z_{\mathfrak{t p}} & =l_{1} \sin \theta_{2}+l_{2} \sin \left(\theta_{2}+\theta_{3}\right)+\cdots+l_{n} \sin \left(\theta_{2}+(n-1) \theta_{3}\right), \\
& =\sum_{k=1}^{n} l_{k} \sin \left[\theta_{2}+(k-1) \theta_{3}\right] .
\end{aligned}
$$

The inverse kinematics of this manipulator are approximated using numerical iterations such as Newton-Raphson, because we have only three equations and three unknown variables, $\theta_{1}, \theta_{2}, \theta_{3}$. The next section shows the crippling effect of each joint on the workspace of the manipulator.

\section{Crippling of the manipulator}

\subsection{Crippling of $\theta_{1}$ joint}

To enable the planar manipulator capable of moving in a three dimensional workspace, the angle $\theta_{1}$ controls the rotation of the entire manipulator around the vertical axis, as shown in Figure 2. Therefore, when $\theta_{1}$ has a value other than the desired one, it is impossible to attain the desired target point. From equation (3) it is seen that the $\boldsymbol{x}$ and $\boldsymbol{y}$ axes of the target point are functions of $\theta_{1}$, which means that when this angle has an incorrect value because of crippling, it will be impossible to attain the target point. 


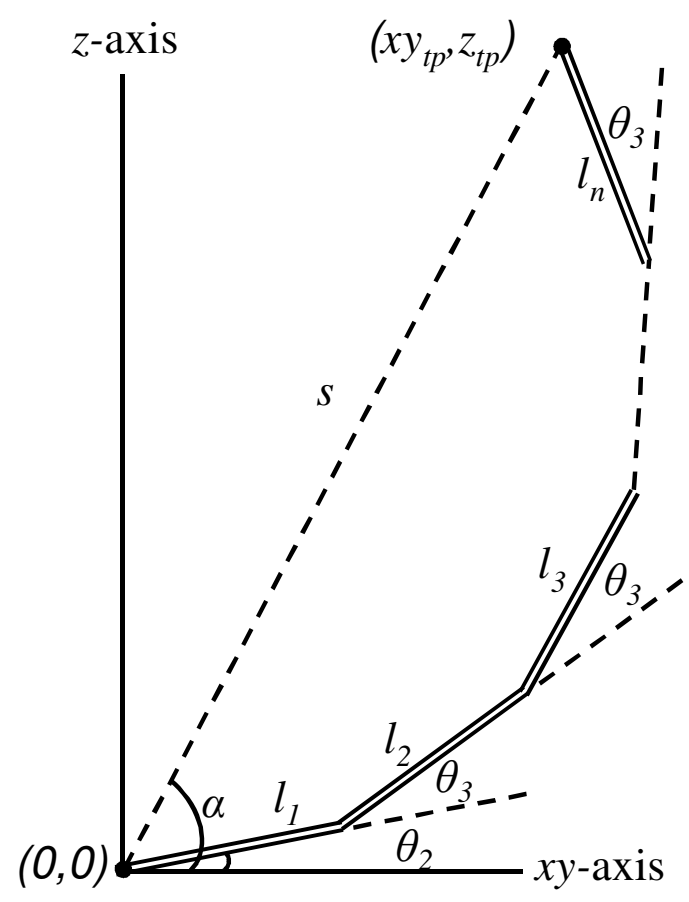

Figure 3: The configuration of the planar manipulator using the method of Yahya et al. [8]

\subsection{Crippling of $\theta_{2}$ joint}

As long as the $\theta_{1}$ joint is uncrippled, the manipulator freely rotates in a three dimensional workspace. Therefore, we concentrate on the planar manipulator which moves in the $x y-z$ plane, as shown in Figure 3.

For this manipulator, we define the target point by its distance $s$ from the origin and the angle $\alpha$ shown in Figure 3. This distance is

$$
s=\sqrt{x_{\mathrm{tp}}^{2}+y_{\mathrm{tp}}^{2}+z_{\mathrm{tp}}^{2}} .
$$

The value of $\mathrm{s}$ is not a function of $\theta_{2}$; as shown in Figure 4, changing the 


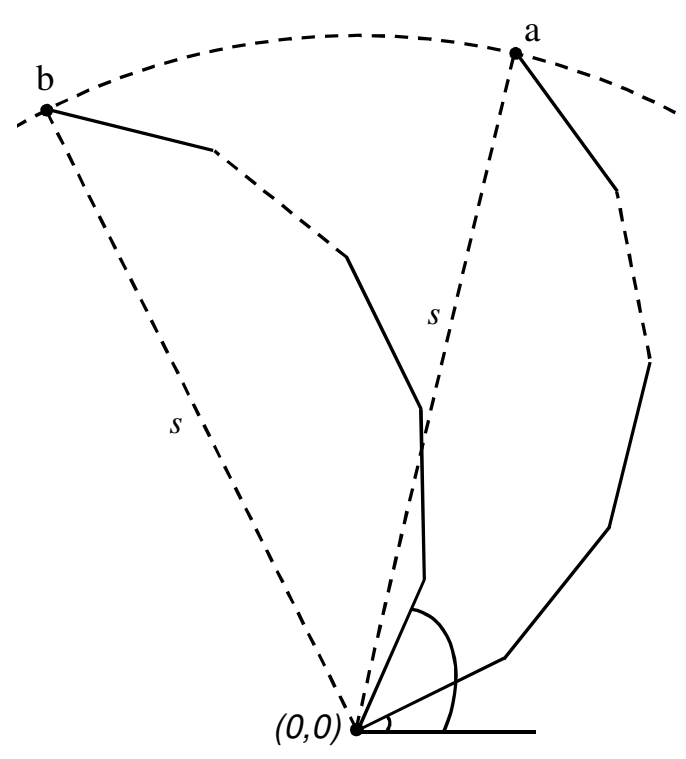

Figure 4: Affect of modifying $\theta_{2}$ on the angle $\alpha$.

value of $\theta_{2}$ will change the the angle $\alpha$ but not the distance $s$. To prove this mathematically, $s$ is calculated using the right angled triangle shown in Figure 5, and equation (4) is rewritten as

$$
s=\sqrt{\left(\sum_{k=1}^{n} l_{k} \cos \left[(k-1) \theta_{3}\right]\right)^{2}+\left(\sum_{k=2}^{n} l_{k} \sin \left[(k-1) \theta_{3}\right]\right)^{2}}
$$

which is not a function of $\theta_{2}$.

The angle of the target point is

$$
\alpha=\tan ^{-1}\left(\sum_{k=1}^{n} l_{k} \cos \left[\theta_{2}+(k-1) \theta_{3}\right]\right)\left(\sum_{k=1}^{n} l_{k} \sin \left[\theta_{2}+(k-1) \theta_{3}\right]\right)^{-1} .
$$

This equation shows that $\alpha$ is a function of $\theta_{2}$. When the joint $\theta_{2}$ is inoperable, if the angles between the links are equal $\left(\theta_{3}=\theta_{4}=\cdots=\theta_{n+1}\right)$, then changing 


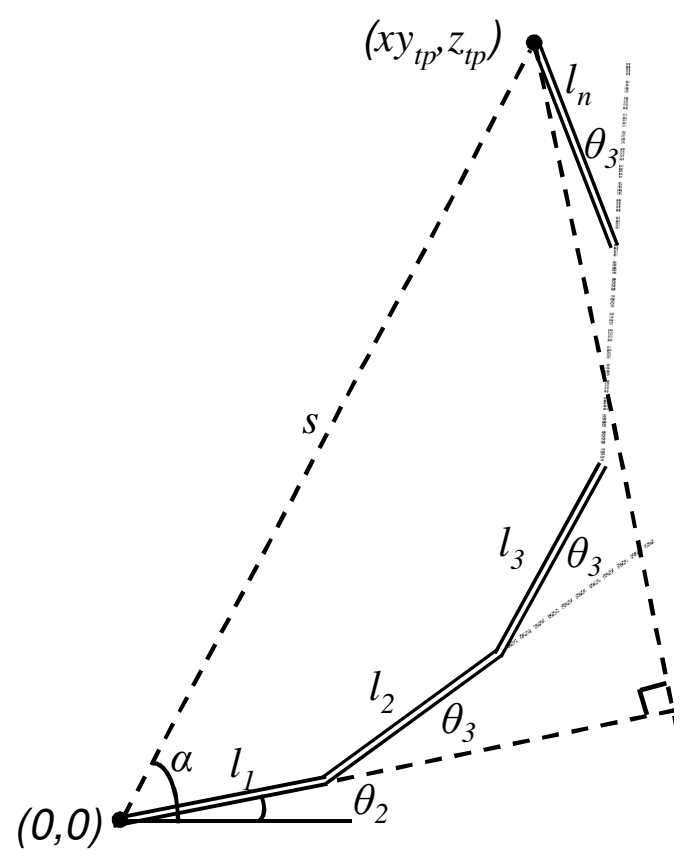

Figure 5: Calculating distance $s$ without using angle $\theta_{2}$.

angle $\theta_{3}$ is not sufficient to allow the end-effector to reach the target point. For example, to move the end-effector from point $a$ to point $b$ (as shown in Figure 4), which maintain the same distance from the origin, $\theta_{2}$ must change. In contrast, changing $\theta_{3}$ without changing $\theta_{2}$ will move the end-effector along a path which has different values of both $s$ and $\alpha$, as shown in Figure 6.

\subsection{Crippling of $\theta_{3}$ joint}

From equation (5), the distance $s$ between the origin and the end-effector is a function of the joint angle $\theta_{3}$. This means that when the joint $\theta_{3}$ is inoperable, there is no way to move the end-effector to the target point, unless it lies on a sphere of radius s. In another words, when the joint $\theta_{3}$ becomes 


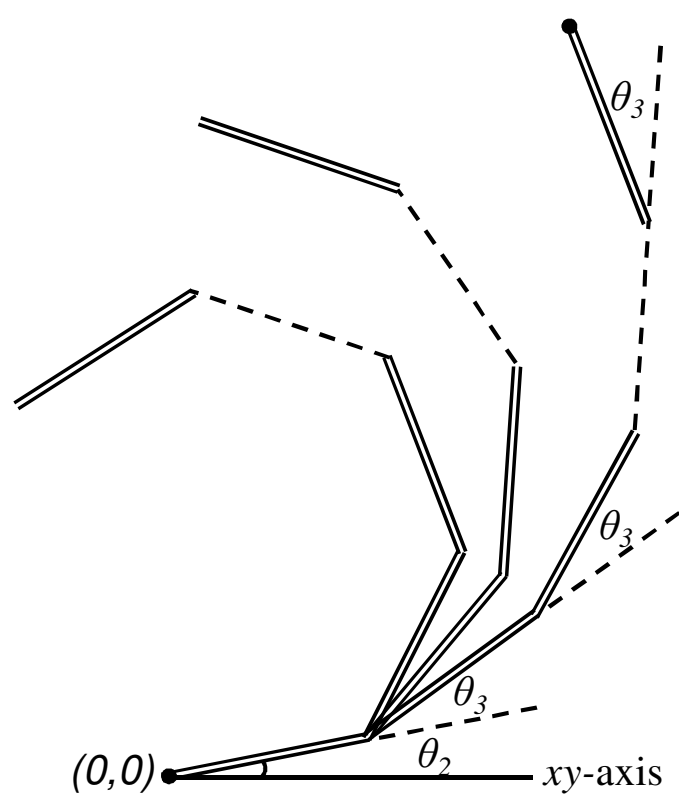

Figure 6: Effect of modifying $\theta_{3}$ without changing $\theta_{2}$.

inoperable, then the distance $s$ is fixed, no matter what the values of the angles $\theta_{1}$ and $\theta_{2}$.

\subsection{Crippling of $\theta_{4}, \theta_{5}, \ldots, \theta_{\mathfrak{n}}$ joint}

Now consider a breakdown of joint $\theta_{4}$. Provided $\theta_{4}=\theta_{5}=\cdots=\theta_{n+1}$, all these joints will be inoperable and will act as one link only, as shown in Figure 7. In this figure, $\theta_{4}=\theta_{5}=\cdots=\theta_{n+1}=\theta_{\text {io }}$, where $\theta_{\text {io }}$ is the value of the joint $\theta_{4}$ when it becomes inoperable and is not equal to $\theta_{3}$. For this 


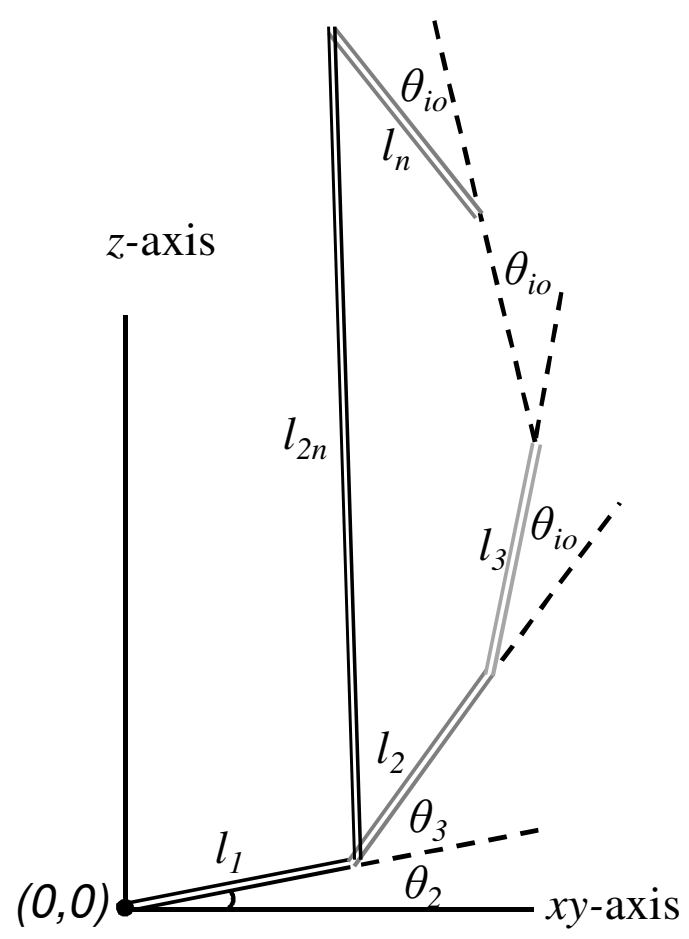

Figure 7: Crippling of joint $\theta_{4}$.

manipulator, the target point coordinates are

$$
\begin{aligned}
& x_{\mathrm{tp}}=\cos \theta_{1}\left(l_{1} \cos \theta_{2}+\sum_{\mathrm{k}=2}^{n} l_{k} \cos \left[\theta_{2}+\theta_{3}+(k-2) \theta_{\mathrm{io}}\right]\right), \\
& y_{\mathrm{tp}}=\sin \theta_{1}\left(l_{1} \cos \theta_{2}+\sum_{\mathrm{k}=2}^{n} l_{\mathrm{k}} \cos \left[\theta_{2}+\theta_{3}+(k-2) \theta_{\mathrm{io}}\right]\right), \\
& z_{\mathrm{tp}}=l_{1} \sin \theta_{2}+\sum_{\mathrm{k}=2}^{n} l_{k} \sin \left[\theta_{2}+\theta_{3}+(k-2) \theta_{\mathrm{io}}\right] .
\end{aligned}
$$

To calculate whether there is a solution for the inverse kinematics of this 
manipulator, the Newton-Raphson approximations are used, because we have three equations and three unknown variables $\left(\theta_{1}, \theta_{2}, \theta_{3}\right)$. From Figure 7 ,

$$
l_{2 n}=\sqrt{\left(\sum_{k=2}^{n} l_{k} \cos \left[\theta_{3}+(k-2) \theta_{i o}\right]\right)^{2}+\left(\sum_{k=2}^{n} l_{k} \sin \left[\theta_{3}+(k-2) \theta_{i o}\right)^{2}\right.} .
$$

where $\mathrm{n}$ is the number of links.

Now, if the breakdown occurs at joint $\theta_{5}$, then an equation similar to equation (7) determines whether there is solution for the inverse kinematics of the manipulator. The only change in these coordinate equations is that two joints move with angle $\theta_{3}$ instead of one and $(n-3)$ joints move with angle $\theta_{\text {io }}$ instead of $(n-2)$. Using the same concept, equation (7) is used to calculate the inverse kinematics when joints $\theta_{6}, \ldots, \theta_{n+1}$ become inoperable.

\section{Simulation results}

Consider the six degrees of freedom manipulator of Figure 2. We only consider the five links planar manipulator, $n=5$. When there is no breakdown, the maximum reach of the manipulator is

$$
R_{o}=l_{1}+l_{2}+l_{3}+l_{4}+l_{5} .
$$

If the manipulator is equipped with one passive joint $p$, which is kept locked during manipulation tasks, then the degrees of freedom of the manipulator are reduced and the workspace is also reduced. The workspace of the underactuated manipulator is an annulus with inner and outer radii $R_{i}\left(\theta_{p}\right)$ and $R_{o}\left(\theta_{p}\right)$, respectively. The workspace area is

$$
A_{p}\left(\theta_{p}\right)=\pi\left[R_{o}^{2}\left(\theta_{p}\right)-R_{i}^{2}\left(\theta_{p}\right)\right] .
$$

We now discuss the boundary radii and workspace area calculations for all possible inoperable joints on the six degrees of freedom manipulator of Figure 2. 


\subsection{Breakdown of $\theta_{1}$ joint}

As mentioned in Section 3.1, when $\theta_{1}$ has a value other than the desired one, it is impossible to attain the specified target point because the manipulator loses its ability to move in three dimensions and becomes just a planar manipulator with a circular workspace. As explained in Section 3.2, the distance $\mathbf{s}$ is not a function of $\theta_{2}$ but a function of $\theta_{3}$ only. The end-effector of the manipulator is at its maximum position when it is fully stretched out, that is, when the angle $\theta_{3}$ is zero. In this case the distance $s$ is equal to the sum of all lengths of links of the manipulator.

The inner and outer reach of the manipulator is calculated from equation (5) with $n=5$ :

$$
\begin{aligned}
s^{2}= & {\left[l_{1}+l_{2} \cos \left(\theta_{3}\right)+l_{3} \cos \left(2 \theta_{3}\right)+l_{4} \cos \left(3 \theta_{3}\right)+l_{5} \cos \left(4 \theta_{3}\right)\right]^{2} } \\
& +\left[l_{1}+l_{2} \sin \left(\theta_{3}\right)+l_{3} \sin \left(2 \theta_{3}\right)+l_{4} \sin \left(3 \theta_{3}\right)+l_{5} \sin \left(4 \theta_{3}\right)\right]^{2} .
\end{aligned}
$$

To find the values of $\theta_{3}$ which minimise or maximise $s$, we take the derivative of the above equation with respect to $\theta_{3}$ :

$$
\begin{aligned}
2 s \frac{d s}{d \theta_{3}}= & 2\left[l_{1}+l_{2} \cos \left(\theta_{3}\right)+l_{3} \cos \left(2 \theta_{3}\right)+l_{4} \cos \left(3 \theta_{3}\right)+l_{5} \cos \left(4 \theta_{3}\right)\right] \\
& \times\left[-l_{2} \sin \left(\theta_{3}\right)-2 l_{3} \sin \left(2 \theta_{3}\right)-3 l_{4} \sin \left(3 \theta_{3}\right)-4 l_{5} \sin \left(4 \theta_{3}\right)\right] \\
& +2\left[l_{2} \sin \left(\theta_{3}\right)+l_{3} \sin \left(2 \theta_{3}\right)+l_{4} \sin \left(3 \theta_{3}\right)+l_{5} \sin \left(4 \theta_{3}\right)\right] \\
& \times\left[l_{2} \cos \left(\theta_{3}\right)+2 l_{3} \cos \left(2 \theta_{3}\right)+3 l_{4} \cos \left(3 \theta_{3}\right)+4 l_{5} \cos \left(4 \theta_{3}\right)\right] .
\end{aligned}
$$

Now, the values of $\theta_{3}$ that make $s$ minimum or maximum are calculated by setting $\mathrm{ds} / \mathrm{d} \theta_{3}$ to zero. The next step is to substitute these values of $\theta_{3}$ into equation (5) or (11) to find the minimum and maximum reach.

\subsection{Breakdown of $\theta_{2}$ joint}

When a breakdown occurs on the $\theta_{2}$ joint, the manipulator is reduced, in practice, to a four link underactuated manipulator, and the origin is shifted 
to the joint between link one and link two. We calculate the distance from the new origin to the end-effector from

$$
\begin{aligned}
s=\{ & {\left[l_{2} \cos \left(\theta_{3}\right)+l_{3} \cos \left(2 \theta_{3}\right)+l_{4} \cos \left(3 \theta_{3}\right)+l_{5} \cos \left(4 \theta_{3}\right)\right]^{2} } \\
& \left.+\left[l_{2} \sin \left(\theta_{3}\right)+l_{3} \sin \left(2 \theta_{3}\right)+l_{4} \sin \left(3 \theta_{3}\right)+l_{5} \sin \left(4 \theta_{3}\right)\right]^{2}\right\}^{1 / 2} .
\end{aligned}
$$

Again, the values of $\theta_{3}$ which minimise or maximise $s$ are calculated by setting $\mathrm{ds} / \mathrm{d} \theta_{3}$ to zero in

$$
\begin{aligned}
2 s \frac{d s}{d \theta_{3}}= & 2\left[l_{2} \cos \left(\theta_{3}\right)+l_{3} \cos \left(2 \theta_{3}\right)+l_{4} \cos \left(3 \theta_{3}\right)+l_{5} \cos \left(4 \theta_{3}\right)\right] \\
& \times\left[-l_{2} \sin \left(\theta_{3}\right)-2 l_{3} \sin \left(2 \theta_{3}\right)-3 l_{4} \sin \left(3 \theta_{3}\right)-4 l_{5} \sin \left(4 \theta_{3}\right)\right] \\
& +2\left[l_{2} \sin \left(\theta_{3}\right)+l_{3} \sin \left(2 \theta_{3}\right)+l_{4} \sin \left(3 \theta_{3}\right)+l_{5} \sin \left(4 \theta_{3}\right)\right] \\
& \times\left[l_{2} \cos \left(\theta_{3}\right)+2 l_{3} \cos \left(2 \theta_{3}\right)+3 l_{4} \cos \left(3 \theta_{3}\right)+4 l_{5} \cos \left(4 \theta_{3}\right)\right],
\end{aligned}
$$

and then substituting the solution of $\theta_{3}$ into equation (13).

\subsection{Breakdown of $\theta_{3}$ joint}

Because the motor which controls the $\theta_{3}$ joint is the same motor which controls all higher joints $\left(\theta_{4}, \theta_{5}, \theta_{6}\right)$, any breakdown of the $\theta_{3}$ joint means that these higher joints are also inoperable. In other words, when joint three becomes passive, $\theta_{3}=\theta_{i o}$, the manipulator is reduced to a one link underactuated mechanism with distance from origin to end-effector

$$
\begin{aligned}
s=\{ & {\left[l_{1}+l_{2} \cos \left(\theta_{\text {io }}\right)+l_{3} \cos \left(2 \theta_{\text {io }}\right)+l_{4} \cos \left(3 \theta_{\text {io }}\right)+l_{5} \cos \left(4 \theta_{\text {io }}\right)\right]^{2} } \\
& \left.+\left[l_{2} \sin \left(\theta_{\text {io }}\right)+l_{3} \sin \left(2 \theta_{\text {io }}\right)+l_{4} \sin \left(3 \theta_{\text {io }}\right)+l_{5} \sin \left(4 \theta_{\text {io }}\right)\right]^{2}\right\}^{1 / 2} .
\end{aligned}
$$




\subsection{Breakdown of $\theta_{4}$ joint}

When a breakdown occurs in the $\theta_{4}$ joint the manipulator is reduced, in practice, to a two link underactuated mechanism with link lengths $l_{1}$ and

$$
\begin{aligned}
& l_{25}=\left\{\left[l_{2} \cos \left(\theta_{3}\right)+l_{3} \cos \left(\theta_{3}+\theta_{\text {io }}\right)+l_{4} \cos \left(\theta_{3}+2 \theta_{\text {io }}\right)+l_{5} \cos \left(\theta_{3}+3 \theta_{\text {io }}\right)\right]^{2}\right. \\
& \left.+\left[l_{2} \sin \left(\theta_{3}\right)+l_{3} \sin \left(\theta_{3}+\theta_{\text {io }}\right)+l_{4} \sin \left(\theta_{3}+2 \theta_{\text {io }}\right)+l_{5} \sin \left(\theta_{3}+3 \theta_{\text {io }}\right)\right]^{2}\right\}^{1 / 2},
\end{aligned}
$$

where $\theta_{4}=\theta_{\text {io }}$. Therefore, the inner and outer radii are

$$
R_{i}=\left|l_{1}-l_{25}\right| \text { and } R_{o}=l_{1}+l_{25} .
$$

From these two radii, the area of the workspace is

$$
\mathrm{V}_{\mathrm{p}}=4 \pi l_{1} l_{25} .
$$

\subsection{Breakdown of $\theta_{5}$ joint}

When the $\theta_{5}$ joint becomes inoperable, the manipulator is reduced, in practice, to a three link underactuated mechanism with link lengths $l_{1}, l_{2}$ and

$$
\begin{aligned}
l_{35}=\{ & {\left[l_{3} \cos \left(\theta_{3}\right)+l_{4} \cos \left(\theta_{3}+\theta_{\text {io }}\right)+l_{5} \cos \left(\theta_{3}+2 \theta_{\text {io }}\right)\right]^{2} } \\
& \left.+\left[l_{3} \sin \left(\theta_{3}\right)+l_{4} \sin \left(\theta_{3}+\theta_{\text {io }}\right)+l_{5} \sin \left(\theta_{3}+2 \theta_{\text {io }}\right)\right]^{2}\right\}^{1 / 2},
\end{aligned}
$$

where $\theta_{5}=\theta_{\text {io }}$. As mentioned previously, $\theta_{\text {io }}$ is constant and equal to the angle at which the joint breaks down. The distance from the origin to the end-effector is

$$
\begin{aligned}
s=\{ & {\left[l_{1} \cos \left(\theta_{2}\right)+l_{2} \cos \left(\theta_{2}+\theta_{3}\right)+l_{3} \cos \left(\theta_{2}+2 \theta_{3}\right)\right.} \\
& \left.+l_{4} \cos \left(\theta_{2}+2 \theta_{3}+\theta_{\text {io }}\right)+l_{5} \cos \left(\theta_{2}+2 \theta_{3}+2 \theta_{\text {io }}\right)\right]^{2} \\
& {\left[l_{1} \sin \left(\theta_{2}\right)+l_{2} \sin \left(\theta_{2}+\theta_{3}\right)+l_{3} \sin \left(\theta_{2}+2 \theta_{3}\right)\right.} \\
& \left.\left.+l_{4} \sin \left(\theta_{2}+2 \theta_{3}+\theta_{\text {io }}\right)+l_{5} \sin \left(\theta_{2}+2 \theta_{3}+2 \theta_{\text {io }}\right)\right]^{2}\right\}^{1 / 2} .
\end{aligned}
$$


We need to calculate the values of $\theta_{3}$ which minimise or maximise the distance $s$, which means calculating the inner and outer radii of the workspace. To calculate the distance $s$, we determine $\theta_{3}$ when $\mathrm{ds} / \mathrm{d} \theta_{3}$ is set to zero, and then substitute these values of $\theta_{3}$ into equation (20). The derivative of equation (20) is

$$
\begin{aligned}
2 s \frac{d s}{d \theta_{3}}= & 2\left[l_{1} \cos \left(\theta_{2}\right)+l_{2} \cos \left(\theta_{2}+\theta_{3}\right)+l_{3} \cos \left(\theta_{2}+2 \theta_{3}\right)\right. \\
& \left.+l_{4} \cos \left(\theta_{2}+2 \theta_{3}+\theta_{\text {io }}\right)+l_{5} \cos \left(\theta_{2}+2 \theta_{3}+2 \theta_{\text {io }}\right)\right] \\
& \times\left[-l_{2} \sin \left(\theta_{2}+\theta_{3}\right)-2 l_{3} \sin \left(\theta_{2}+2 \theta_{3}\right)\right. \\
& \left.-2 l_{4} \sin \left(\theta_{2}+2 \theta_{3}+\theta_{\text {io }}\right)-2 l_{5} \sin \left(\theta_{2}+2 \theta_{3}+2 \theta_{\text {io }}\right)\right] \\
& +2\left[l_{1} \sin \left(\theta_{2}\right)+l_{2} \sin \left(\theta_{2}+\theta_{3}\right)+l_{3} \sin \left(\theta_{2}+2 \theta_{3}\right)\right. \\
& \left.+l_{4} \sin \left(\theta_{2}+2 \theta_{3}+\theta_{\text {io }}\right)+l_{5} \sin \left(\theta_{2}+2 \theta_{3}+2 \theta_{\text {io }}\right)\right] \\
& \times\left[l_{2} \cos \left(\theta_{2}+\theta_{3}\right)+2 l_{3} \cos \left(\theta_{2}+2 \theta_{3}\right)\right. \\
& \left.+2 l_{4} \cos \left(\theta_{2}+2 \theta_{3}+\theta_{\text {io }}\right)+2 l_{5} \cos \left(\theta_{2}+2 \theta_{3}+2 \theta_{\text {io }}\right)\right] .
\end{aligned}
$$

\subsection{Breakdown of $\theta_{6}$ joint}

When the $\theta_{6}$ joint becomes inoperable, the manipulator is reduced, in practice, to a four link underactuated mechanism. The link lengths of this manipulator are $l_{1}, l_{2}, l_{3}$ and

$$
l_{45}=\sqrt{l_{4}^{2}+l_{5}^{2}+2 l_{4} l_{5} \cos \theta_{\text {io }}},
$$

where $\theta_{6}=\theta_{i o}$. The distance from the origin to the end-effector is

$$
\begin{aligned}
s= & \left\{\left[l_{1} \cos \left(\theta_{2}\right)+l_{2} \cos \left(\theta_{2}+\theta_{3}\right)+l_{3} \cos \left(\theta_{2}+2 \theta_{3}\right)\right\}\right. \\
& \left.+l_{4} \cos \left(\theta_{2}+3 \theta_{3}\right)+l_{5} \cos \left(\theta_{2}+3 \theta_{3}+\theta_{i o}\right)\right]^{2} \\
& +\left[l_{1} \sin \left(\theta_{2}\right)+l_{2} \sin \left(\theta_{2}+\theta_{3}\right)+l_{3} \sin \left(\theta_{2}+2 \theta_{3}\right)\right. \\
& \left.\left.+l_{4} \sin \left(\theta_{2}+3 \theta_{3}\right)+l_{5} \sin \left(\theta_{2}+3 \theta_{3}+\theta_{\text {io }}\right)\right]^{2}\right\}^{1 / 2} .
\end{aligned}
$$


To calculate the minimum and maximum distances $s$, we set $\mathrm{ds} / \mathrm{d} \theta_{3}$ to zero and determine $\theta_{3}$, then we substitute these values of $\theta_{3}$ into equation (23). The derivative of equation (23) is

$$
\begin{aligned}
2 s \frac{d s}{d \theta_{3}}= & 2\left[l_{1} \cos \left(\theta_{2}\right)+l_{2} \cos \left(\theta_{2}+\theta_{3}\right)+l_{3} \cos \left(\theta_{2}+2 \theta_{3}\right)\right. \\
& \left.+l_{4} \cos \left(\theta_{2}+3 \theta_{3}\right)+l_{5} \cos \left(\theta_{2}+3 \theta_{3}+\theta_{\text {io }}\right)\right] \\
& \times\left[-l_{2} \sin \left(\theta_{2}+\theta_{3}\right)-2 l_{3} \sin \left(\theta_{2}+2 \theta_{3}\right)\right. \\
& \left.-3 l_{4} \sin \left(\theta_{2}+3 \theta_{3}\right)-3 l_{5} \sin \left(\theta_{2}+3 \theta_{3}+\theta_{\text {io }}\right)\right] \\
& +2\left[l_{1} \sin \left(\theta_{2}\right)+l_{2} \sin \left(\theta_{2}+\theta_{3}\right)+l_{3} \sin \left(\theta_{2}+2 \theta_{3}\right)\right. \\
& \left.+l_{4} \sin \left(\theta_{2}+3 \theta_{3}\right)+l_{5} \sin \left(\theta_{2}+3 \theta_{3}+\theta_{\text {io }}\right)\right] \\
& \times\left[l_{2} \cos \left(\theta_{2}+\theta_{3}\right)+2 l_{3} \cos \left(\theta_{2}+2 \theta_{3}\right)\right. \\
& \left.+3 l_{4} \cos \left(\theta_{2}+3 \theta_{3}\right)+3 l_{5} \cos \left(\theta_{2}+3 \theta_{3}+\theta_{\text {io }}\right)\right] .
\end{aligned}
$$

\section{Conclusion}

We assessed whether, in the event of crippling, one might exploit the remaining degrees of freedom of a robot to keep it in service, pending repair. A six degrees of freedom three dimensional manipulator was used for simulation. The results showed that when any one of the first three joints becomes inoperable, the end-effector is unable to attain all target points. In contrast, for the $\mathrm{n}=5$ case, when any of the last three joints breakdown, the operable joints still enable the end-effector to attain the target point. The inner and outer radii of the workspace, attainable with one inoperable joint, were calculated and discussed. 


\section{References}

[1] X. Xin, J.-H. She, T. Yamasaki and Y. Liu, Swing-up control based on virtual composite links for n-link underactuated robot with passive first joint, Automatica, 45(9):1986-1994, 2009. doi:10.1016/j.automatica.2009.04.023 C417

[2] M. Bergerman and Y. Xu, Dexterity of underactuated manipulators, ICAR, 719-724, 1997. doi:10.1109/ICAR.1997.620261 C418

[3] S, Yahya, M. Moghavvemi and H. A. F. Mohamed, Singularity Avoidance of a Six Degrees of Freedom Three Dimensional Redundant Planar Manipulator, Comput. Math. Appl., 64(5):856-868, 2012. doi:10.1016/j.camwa.2011.12.073 C418, C419, C420

[4] S. Yahya, M. Moghavvemi and H. A. F. Almurib, Joint Torque Reduction of a Three Dimensional Redundant Planar Manipulator, Sensors, 12(6):6869-6892, 2012. doi:10.3390/s120606869 C418, C419, C420

[5] A. K. Pradeep, P. J. Yoder, R. Mukundan, R. J. Schilling, Crippled motion in robots, IEEE T. Aero. Elec. Sys., 24(1):2-13, 1988. doi:10.1109/7.1030 C418

[6] R. G. Roberts, Quantifying the local fault tolerance of a kinematically redundant manipulator, Amer. Contr. Conf., 3:1889-1893, 1995. doi:10.1109/ACC.1995.531215 C418

[7] R. G. Roberts and A. A. Maciejewski. A local measure of fault tolerance for kinematically redundant manipulators, IEEE Int. Conf. Robot., 12(4):543-552, 1996. doi:10.1109/70.508437 C418, C420, C421

[8] S. Yahya, M. Moghavvemi and H. A. F. Mohamed. Geometrical approach of planar hyper-redundant manipulators: Inverse kinematics, path planning and workspace, Simul. Model. Pract. Th., 19(1):406-422, 2011. doi:10.1016/j.simpat.2010.08.001 C420, C421, C422, C424 
[9] H. A. F. Mohamed, S. Yahya, M. Moghavvemi and S. S. Yang. A New Inverse Kinematics Method for Three Dimensional Redundant Manipulators, ICCAS-SICE, 1557-1562, 2009.

http://ieeexplore.ieee.org/xpl/articleDetails.j.sp?arnumber= 5335259\&ref inements\%3D4274859776\%26sortType\%3Dasc_p_ Sequence $\% 26$ filter $\% 3 D A N D \% 28$ p_IS_Number $\% 3 A 5332438 \% 29$

\section{Author addresses}

1. Samer Yahya, Department of Mechanical, Materials and Manufacturing Engineering The University of Nottingham Malaysia Campus, Malaysia mailto:Samer. Yahya@Nottingham. edu.my

2. Haider Abbas F. Mohamed Almurib, Department of Electrical and Electronic Engineering The University of Nottingham Malaysia Campus, Malaysia

3. M. Moghavvemi, Center of Research in Applied Electronics (CRAE) University of Malaya, Malaysia 